\title{
An agent-based computational approach for urban traffic regulation
}

\author{
Neïla Bhouri · Flavien Balbo · Suzanne Pinson
}

Received: 25 July 2011 / Accepted: 26 February 2012 / Published online: 26 May 2012

(C) Springer-Verlag 2012

\begin{abstract}
This paper proposes a bimodal urban traffic control strategy based on a multi-agent model. We call bimodal traffic, a traffic which takes into account both private vehicles and public vehicles such as buses. The objective of this research is to improve global traffic, to reduce bus delays and to improve bus regularity in congested areas of the network. In our agent-based approach, traffic regulation is obtained thanks to communication, collaboration and negotiation between heterogeneous agents. An important feature of our system is that it allows regulation at two levels: macroscopic and microscopic levels. To model in depth regulation procedures, we have introduced special features such as priority levels for buses, computation and update of traffic signal plans, urgency index of intersection stages depending on the level of congestion on the arcs. We have tested our strategy on a small network of six intersections, using the JADE platform. The simulation is described and preliminary results are presented. They show that our MAS strategy improves bus travel time while improving also private vehicles' travel time, decreases bus delays and improves its regularity compared to a classical strategy called fixed-time control strategy.
\end{abstract}

Keywords Urban traffic control · Multi-agent modeling · Public transport · Traffic light . Communication $\cdot$ Collaboration $\cdot$ Negotiation

\section{N. Bhouri $(\bowtie) \cdot$ F. Balbo}

IFSTTAR, GRETTIA, Université Paris Est, "Le Descartes 2"

2 rue de la Butte Verte, 93166 Noisy Le Grand Cedex, France

e-mail: neila.bhouri@ifsttar.fr

F. Balbo $\cdot$ S. Pinson

CNRS, LAMSADE, Université Paris-Dauphine, Place du Marechal de Lattre de Tassigny, 75775 Paris Cedex 16, France

e-mail: balbo@lamsade.dauphine.fr

S. Pinson

e-mail: pinson@lamsade.dauphine.fr

\section{Introduction}

To improve route times of public surface transportation (bus, tramways, shuttles, etc.), cities often use regulation systems at junctions. These systems are referred as urban transport control (UTC) systems. They have been extended later on to give priority to buses. The aim of these systems is to increase average speed of public transport vehicles as well as private vehicles allowed to cross a junction.

Buses priority control can be operated in a passive or in an active way. Passive way consists of making off-line regulation to improve traffic on roads frequently used by buses. Many systems are equipped with a passive priority module; the most famous one is the TRANSYT system [18]. Active way consists of giving priority to detected buses. It needs real-time detection of buses and real-time modification of traffic lights to take into account information about buses. We can cote TUC [9] and SPPORT [10] or CRONOS [5].

The use of these systems is efficient when traffic is light or when they are used to improve a single congested bus route. However, reducing the time of bus journeys, although very important for operating a route, is not the only factor considered by public transport operators whose obligation is to provide passengers services, e.g. keeping interval between buses.

Classical control theory used to regulate bimodal traffic (public and private vehicles) is confronted with the modeling problem. Traffic flow can be modeled at a microscopic level, i.e. modeling the behavior of each bus or at a macroscopic level, i.e. modeling traffic flows. At microscopic level, TRSS (transportation regulation support systems) were developed to follow a micro-regulation-based approach $[1,2]$. One of the weaknesses of these systems is that private vehicle traffic flow is hardly taken into account. If it 
is taken into account, this is only as an external parameter that modify the route times of buses. Another weakness is that traffic light management which is one of the key factors of traffic jams and bus delays is not included in TRSS systems. Furthermore, microscopic modeling is time-consuming therefore not well adapted to build real time control strategies for wide urban networks. On the contrary, macroscopic modeling, i.e. modeling traffic flows, has the objective to reduce time spent in traffic jams so that buses respect their schedule [4]. We can note that macroscopic representation of buses does not allow more than an indirect consideration of bus intervals. In [14] a hybrid model was used: macroscopic modeling for private vehicles and microscopic modeling for public transport. However, the complexity of real life bimodal traffic shows the limits of these classical modeling approaches.

Our objective is to build a traffic control strategy for bimodal traffic that is able to regulate both private vehicle traffic and public vehicle traffic on a whole network and not only on a single junction taking into account vehicle travel time and bus intervals. We make the hypothesis that public vehicles and private vehicles share the same infrastructure. This means that our proposed strategy can be implemented on real networks without a costly modification of the infrastructure with for example separate lanes for buses. However, we can easily consider a network where there are special lanes for buses as a special case of our strategy where the number of cars in front of buses will be equal to zero.

Multi-agent modeling can give a suitable answer to the weakness highlighted above. Multi-agent paradigm is well adapted to transportation domain study since it facilitates an approach by analogy in a domain where the objective is the management of distributed entities. One advantage of this approach is the explicit representation of processes that it models. By reifying the components of the system to be managed, a multi-agent system allows a better understanding of a complex reality. More precisely, by underlining the components and their links makes it easier to understand regulation processes which in turn facilitates its formalization. A significant amount of research has been devoted to multiagent approach in transportation domain. A good review can be found in [7].

We note that multi-agent systems are increasingly present in the field of traffic regulation [1-3,11-13,15-17] and [19]. The problem of traffic lights coordination on the thoroughfares of the route network has been studied in $[6,8,12,13]$ and [19]. The regulation system presented in [20] is related to traffic assignment using negotiation between vehicles and junctions. In [6], the authors present agent-based traffic control mechanisms to control both cars and traffic lights. However, these mechanisms do not take into account priority granted to bus on a large network where traffic is dense and where mac- roscopic as well as microscopic regulation has to be taken into account. To answer these shortcomings, we have developed a multi-agent control strategy with special features as multi-level modeling, priority level for buses, urgency index for intersection stages.

Section 2 describes our network model. Section 3 details the four types of agents that we have identified. In Sect. 4, we present the orchestration mechanism between agents and how agent interactions allow modeling the regulation process at a micro and a macro level. A detailed description of agent behavior, their attributes, their objectives, as well as communication and collaboration protocols is given in Sect. 5. Section 6 provides preliminary results of simulation tests carried out on the Jade platform. Finally, we conclude in Sect. 7.

\section{Network modeling}

In our model, the urban network is represented by an oriented graph $G$. The set $I$ represents the intersections (or junctions) and the set $A$ represents the arcs that connect the intersections. Two intersections can be connected by one or several arcs depending on the number of lanes on the thoroughfare.

An arc is characterized by two types of information:

- Static information: its length $l$ (in $\mathrm{m}$ ), its capacity $c$ [the maximum number of vehicles on arc $i$ in private car unit (in pcu)], its saturation output $d$ [the maximum output of exits from the given arc (in pcu/s)].

- Dynamic information: the number $n$ of vehicles on the arc (in pcu), $v$ the average speed of the vehicles (in pcu/s), $b$ the number of buses and $t f$ the time necessary to evacuate the vehicles on the arc.

By pcu, we mean that all vehicles on the arc are converted to their equivalent in private vehicles, for example a bus is 2.3 pcu depending on its length, a truck can be 2.3 or 4 pcu and so on.

An intersection is specified by a set of stages. A stage contains a set $E$ of entering arcs corresponding to compatible streams of vehicles. These streams are compatible because they can safely cross the intersection simultaneously, else they are called antagonistic. A signal cycle is a repetition of the basic series of signal combinations at an intersection; its duration is called time cycle. A stage is a part of the signal cycle, during which one set of streams has right of way. The set of stages represents the configuration of the junction (the permitted movements and turns). Determining the stages is a task executed offline by the traffic experts. In our model, a stage is therefore defined thanks to its states $s t$, its duration $t$ and an index that gives its priority.

Our model is described in $B N F$ notation. 


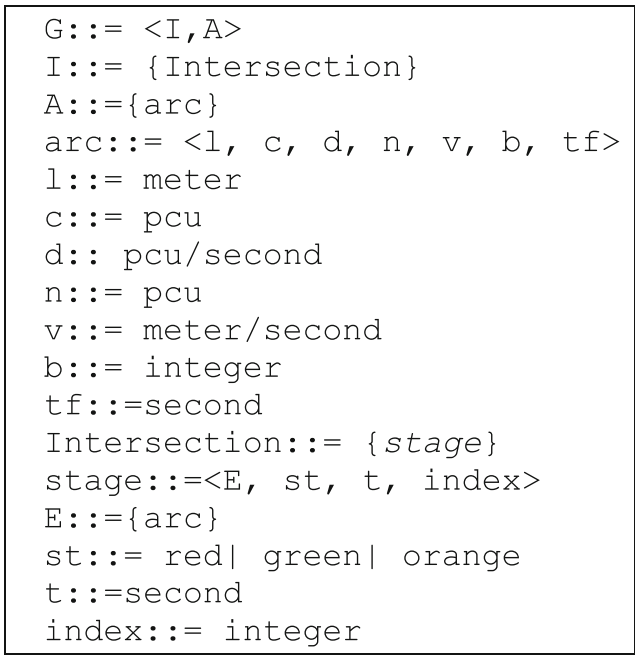

Model 1: Network data model

To represent the different features of a network component, we use pointed notation. For example, the number of buses on arc $i$ belonging to the stage $s a$ is written sa.i.b, with $i \in$ sa.E.

The network is used by a number of bus routes. Each bus route is defined as the number of buses from the same origin and in the same direction, and that service a number of predefined commercial bus stops at regular time intervals. The time spent by a bus at a commercial stop is equal to the pre-set time for passengers to mount, plus additional time to regulate the interval, if required.

In real world, all this information about traffic flow and location of buses is already available in UTC systems since all buses are located in real time and sensors on the road give data about traffic flow. Our proposition could be connected to the UTC system and send commands to traffic lights. Our solution remains scalable because in each intersection, com-

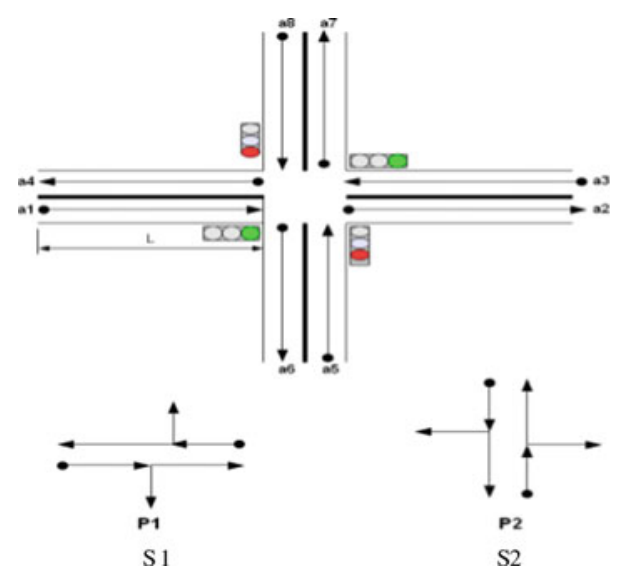

Fig. 1 Example of an intersection with four arcs and two stages S1, S2. S1 allows for the clearing of the arcs a1 and a3, because the entry flows a1 and a 3 can leave the junction at the same green light period. Similarly, S2 clears arcs a5 and a8 putation is done independently of computation done in other intersections.

\section{Agent modeling}

To identify agents and design the MAS, we represent an abstraction of the real system; every entity of the real world is associated to an agent in the virtual world giving a multiagent system (MAS). Each agent is defined in the following way:

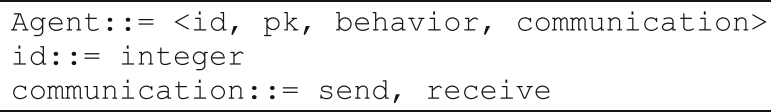

Model 2: Agent generic model

$p k$ and behavior correspond, respectively, to agent private knowledge and agent behaviors; their values are defined for each type of agent, for example, $p k=<E$, st, $t$, index $>$ for a Stage agent (SA). Communication primitives enable agents to communicate. The primitive send (receive) enables an agent to send (to receive) a message. Performatives have the same meaning as FIPA-ACL performatives ${ }^{1}$ (see Model $3)$.

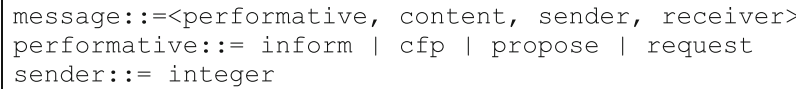

\section{Model 3: Communication model}

The developed MAS is made up of the following agenttypes:

Intersection agent (IA): it is the key agent of our architecture. It is in charge of controlling an intersection with traffic lights, and of developing a traffic signal plan. The intersection agent modifies the planning of the lights according to data sent by approaching buses.

Stage agent: the traffic signal plan is elaborated thanks to the collaboration of the junction and the corresponding stage agents (SAs). Each SA determines the optimal green light split to clear the waiting vehicles on the arcs concerned by the stage. Thus, whatever the complexity of the junction is (and its physical configuration), it is managed by a set of stage agents interacting with the junction agent to develop a plan of actions for the traffic lights.

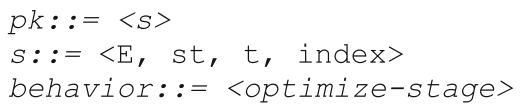

Model 4: Stage agent model

\footnotetext{
${ }^{1}$ http://www.fipa.org.
} 
Bus agent (BA): it represents a bus in the real world. It circulates from one arc (current) to another (next) and communicates with its Bus Route Agent $\left(i d_{B R A}\right)$. For each arc, it has the arc description and the id of the related intersection agents $\left(i d_{I A}\right)$. A bus halts at commercial stops, halts at red lights and obeys the instructions of the BRA (behavior stopRegulation). The objective of each bus agent is to minimize the time spent at traffic lights to minimize journey times (behavior TrafficLightRegulation).

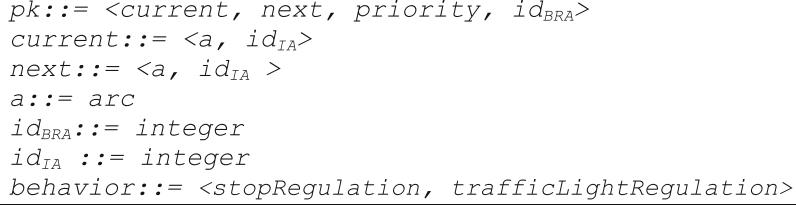

Model 5: Bus agent model

Bus route agent: bus agents can only provide a local view of their environment, more precisely, only the journey covered by the BA. Thus, local optimization carried out by bus agents can have a negative impact on the route, notably on its regularity (i.e. the formation of bus queues or bus gaps). To tackle this problem, we propose an agent who has a global view of the bus agents on the route, and who can control and modify their behavior to guarantee an efficient and regular service.

\section{Multi-agent interaction: the traffic regulation process}

Before getting on the details of the sequence of actions that follows each agent, we present the orchestration mechanism betweens the agents. The four types of agents that we have defined and their interactions allow the modeling of traffic regulation at a micro level as well as at a macro level. As said in our introduction, few research projects have taken into account and have combined in a same system these two levels of modeling. Figure 2 gives a global view of our proposition:

1. Stage agents are based on a macroscopic modeling of the vehicles because each Stage Agent computes the green time it needs and its index of urgency taking into account global traffic flow expressed in pcu.

2. Bus Agents are based on a microscopic modeling of the vehicles. The interaction between the agents related to buses on the network ensures a micro-regulation process. The BRA applies this regulation procedure to each Bus Agent that is late or in advance.

3. The Intersection Agent ensures a macro-micro bimodal regulation process. It computes the plan of traffic lights according to two criteria: (1) the need of the corresponding Stage Agents, which is based on a macroscopic modeling of the global traffic and (2) the priority requests of Bus Agents which are related to a microscopic modeling of buses.

\section{Description of agent behavior}

\subsection{Bus agent}

To minimize the time spent at traffic lights the bus agent interacts with interaction agents and its hierarchical superior agent (BRA). All buses have to provide a regular service and avoid bus queues, in other words, the frequency of buses passing commercial stops must remain stable. To achieve this objective, the BA receives orders from the BRA (for example, stay at the stop for $t \mathrm{~s}$, if the bus is ahead with respect to the position of the preceding bus).

Behavior of a bus agent: Let $\mathrm{t}_{0}$ be the entering time of the bus agent which behaves in the following way:

- When approaching a stop, the BA executes the stopRegulation behavior (see Algorithm 1). It informs the

Fig. 2 Multi-agent interaction

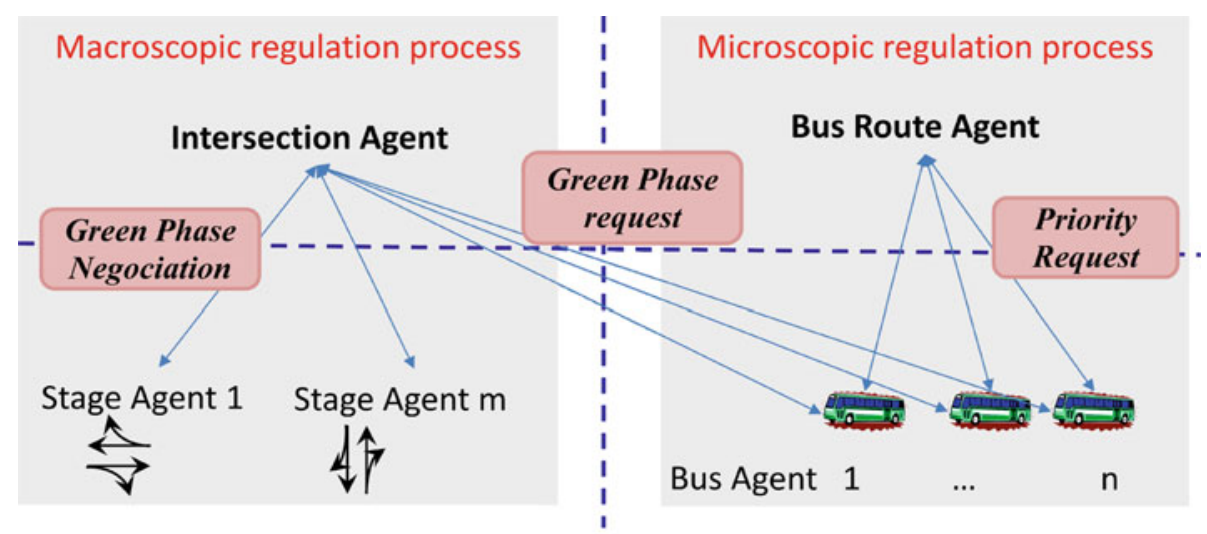

Agent Interaction 
associated BRA whose identification is $i d_{B R A}$. The BRA then calculates the duration of the regulation interval and its level of priority and sends it to the bus agent. Before leaving the stop, the bus must wait during passenger loading time, as well as during the potential regulation time (not taken into account in the algorithm).

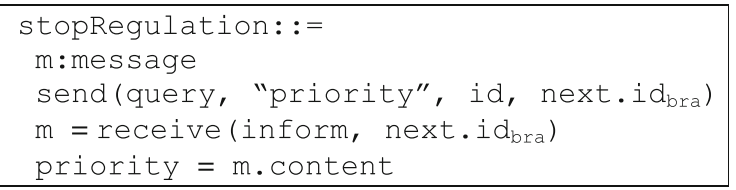

Algorithm 1: Bus agent StopRegulation algorithm

- When approaching a traffic light, the BA executes the trafficLightRegulation behavior (see Algorithm 2). It sends a message to retrieve information from the arc (the number of vehicles that precede it, the length, capacity, and exit output of the arc).

With these data, the BA calculates a time-space request that is transmitted to the IA whose identification is $i d_{I A}$ to prevent an eventual stop at the red light at the following intersection. The IA then attempts to satisfy the demand (see intersection agent below);

Calculation of a green light request. This calculation is specified by the interval of time during which the green light is granted to the actual arc so that the bus can pass without stopping at the next junction. Let $t_{b}$ be the beginning time and $t_{e}=t_{b}+\delta t$ be the ending time of the requested interval ( $\delta t$ is a constant value in $\mathrm{s}$ ). The calculation of $t_{b}$ is carried out as follows: the bus enters the arc and finds in the worst case $n$ vehicles ahead of it, the vehicles move to the traffic lights lane to wait for the green light; the time to evacuate it is $t f$. To continue along its route, the queue of vehicles has to be dispersed before it arrives. The green light should thus be granted at the arc at the time: $t_{b}=t_{0}+l / v-t f$.

The requested interval together with other information (id-number of the bus, its priority, the actual arc of the bus, the next arc to be traveled by the bus) are sent to the IA (at the next intersection) who attempts to modify the plan for the lights to satisfy the request. The Algorithm $2^{2}$ below gives the exact behavior.

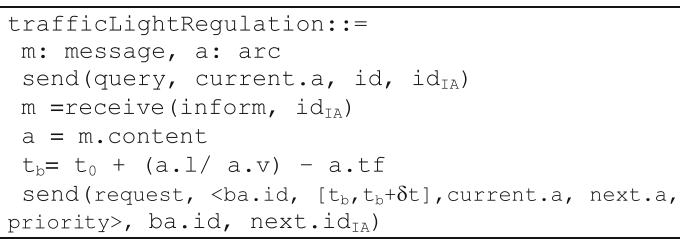

Algorithm 2: Bus agent trafficLightRegulation algorithm

2 Remember that the variables are written with pointed notation. For example m. content means the content of message m, ba.id means the id of bus agent ba, a. 1 means the length of arc a.

\subsection{Intersection agent}

The IA is the key agent of our architecture. The IA supervises the group of stage agents who collaborate together to establish a plan for traffic lights. This plan will, on one hand, maximize the capacity of the junction and, on the other hand, attempt to satisfy, as far as possible, the requested interval of buses. The IA use the static and dynamic data (Model 6).

Static data are the maximum value of the traffic light cycle (TCMAX $=120 \mathrm{~s}$ ). For each cycle, there is an interval of lost time, i.e. the period of orange or all red. The all red light is a period during which all the arcs from the same junction have a red light to clear the centre of the junction and thus prevent accidents. This fixed period, in conformity with the architecture of the junction, does not depend on the length of the cycle. It is fixed here to a 2 -s period after each stage.

Dynamic data are the list of received requested data from Bus agents (see Algorithm 2) that have been processed to find the useful information for the Macro-regulation behavior and the data related to the stages. Each request is specified as follows: $R=<s, t_{b}, t_{e}$, priority $>$, where $s$ is the stage that will allow the passage of the given bus, $t_{b}$ the time when the bus is expected to arrive at the traffic light, $t_{e}$, the time when the rear of the bus leaves the arc, and finally priority is the level of bus priority defined by the BRA (see section below on BRA). Each stage is defined by $i d_{S A}$ related to the intersection, its priority (priority) and the minimum time to evacuate the axes $(t)$.

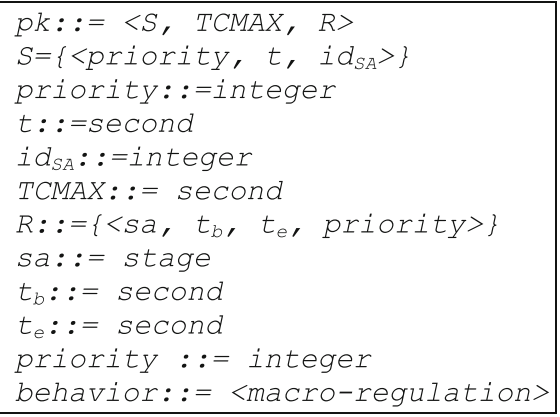

Model 6: Insersection agent model

At the end of each cycle, the IA triggers the process of calculating the traffic signal plan for the given cycle. This plan determines the duration of the green light and the ranking of each stage. When the IA receives a request, it records it in the database. The IA then decides to accept or to refuse this request at time $t_{b}$. As proposed in [21] for conflict resolution in planning, the modification of a traffic signal plan following a priority request by a bus is as follows: (1) extension of a stage (delay or advance), without exceeding the maximal duration of a stage; (2) introduction of a new stage into the plan. 
Calculation of a traffic signal plan The plan (Plan) is calculated through the collaboration of the Intersection agent and the corresponding Stage Agents. The IA plays the role of a manager in supervising the SAs that act as participants (see Algorithm 3).

The IA begins by forming a group of collaborators called collab_group including the list of stage agents that needs to be managed. IA initializes the variable $t c$ that controls the size of the calculated cycle. IA sends a message request to the agents of the collab_group asking them for the time necessary to clear all the vehicles from their stages, beginning at instant $t$. Every agent of the collab_group calculates its desired green light duration and an index that measures the urgency of the stage. It sends these data to the manager (to simplify we suppose that the communication is synchronous) that updates its knowledge about this agent using the update ( $s a$, response. content) procedure and the value of the duration of the proposed cycle $(d=d+$ sa.t $))$.

When all the agents of collab_group have been updated, if $d>t c$ then the manager has to solve a conflict using the conflict-resolution (collab-group, tc) procedure since the size of the cycle exceeds the maximum size. Conflict is solved when $d$ previously calculated becomes less or equal to $t c$. The manager selects the most urgent stage ( $s a=$ argMax(priority, collab_group)). It sends an accept message to the stage agent in charge of operating this stage; it withdraws the corresponding stage agent from collab_group; it updates the variables $t c, t$ and Plan (update(Plan, sa)); finally IA sends a request as long as collab_group is not empty.

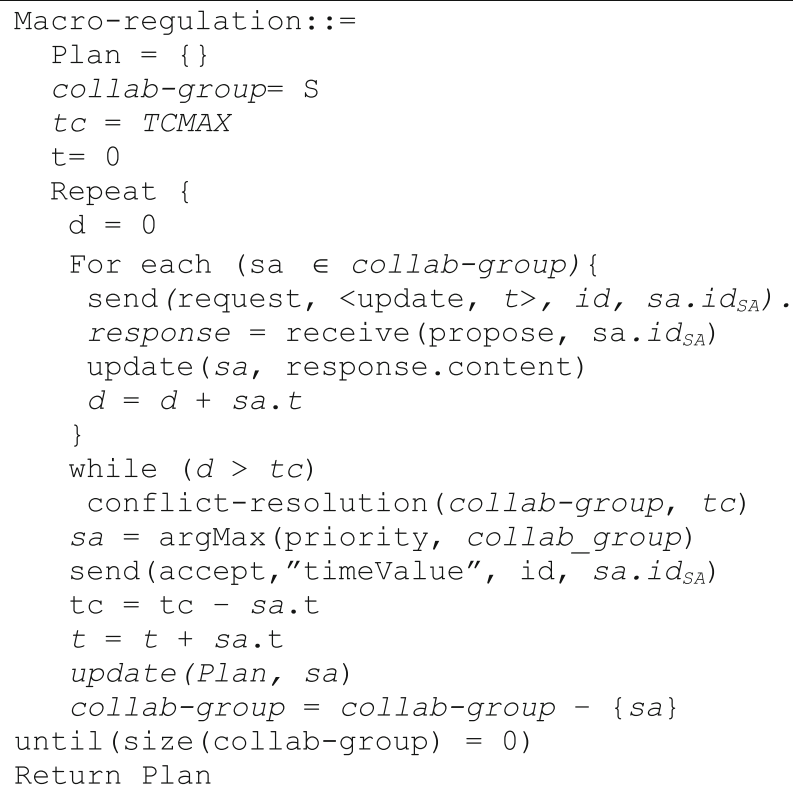

Algorithm 3: Computation of a Plan of light
Conflict resolution. When the sum of green light durations requested by stage agents exceeds the size of the accepted value of the cycle, the IA must restore this sum to the maximal value of the cycle. To achieve a $\Delta t$ reduction, the IA negotiates with the corresponding SAs using a Contract Net Protocol. The cost $c$ of the offer is the number of buses penalized if the stage agent reduces its duration of $\Delta t$, i.e. the number of buses that cannot pass through the intersection during $\Delta \mathrm{t}$.

\subsection{Stage agent}

This agent has a collection of both static and dynamic data as described in Model 1: Network Data Model. They represent its internal state. Static data are the list of entry arcs, the list of arcs authorized to clear if the stage is active (or green)

Dynamic data are related to (1) the state of the stage: active or inactive; (2) the duration of green light attributed to the stage; (3) the starting time of stage execution. 'Active' means that traffic lights controlling the arcs related to this stage are green. Vehicles are therefore authorized to depart.

Behavior of the stage agent The SA participates to the calculation of the traffic signal plan, and is in charge of fixing the optimal duration of green light for the given stage. When the stage agent is asked about the desired duration of green light by the corresponding intersection agent, the duration $\mathrm{d}_{i}$ and an index $\mathrm{I}_{i}$ that measures the urgency of the stage, are computed and transmitted to the intersection agent. If the stage agent receives confirmation from the IA, the stage agent stops the process. If the stage agent receives a $c f p$ (call for propose) with a cost $c$, it computes an offer and sends it to the IA.

Calculation of the desired duration of the green light

The optimal duration of green light is computed by the following formula:

$$
T=\max _{i=1, \ldots, m}\left\{T_{i}\right\} \quad T_{i}=w_{i} \frac{N_{i}}{D_{i}}+\left(1-w_{i}\right) \cdot \frac{N_{i} * L_{i}}{C_{i} * V_{i}}
$$

where $m=|E|$ is the number of entering arcs at the stage, $T_{i}$ the time necessary to clear arc $i, L_{i}$ the length (in m) of arc i, $V_{i}$ the average speed (in $\mathrm{m} / \mathrm{s}$ ) on arc i, $N_{i}$ the number of vehicles expressed in pcu and $D_{i}$ the saturation of arc $i(\mathrm{pcu} / \mathrm{h})$. The first part of the equation $\mathrm{T}_{i}$ expresses the time needed to evacuate the already formed queue at the traffic light and the second term expresses the time needed to evacuate vehicles entering the arc after the beginning of the green, assuming that they arrive at regular intervals.

The optimal duration of green light is computed by the behavior optimize-stage (see Algorithm 4). Its value is the maximum duration to evacuate an arc of the stage.

To award priority to a bus, the urgency index of a stage is defined by the fact that the higher the index, the greater the urgency of the stage. $\mathrm{w}_{i}=n_{i} / c_{i} \in[0,1]$ is a parameter that 
a

b

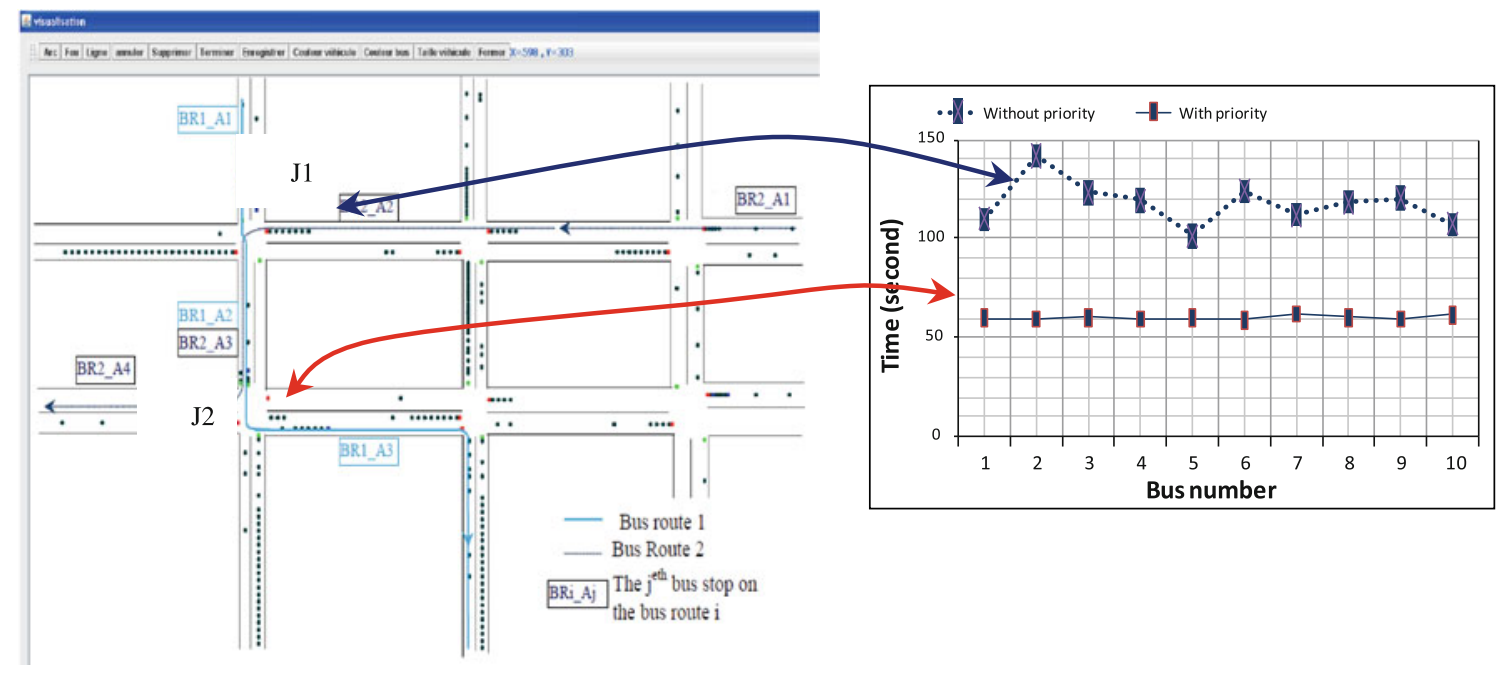

Fig. 3 a The simulated network. b Bus travel time with and without bus priority

indicates the degree of congestion of the arc $i$ and $e$ is the exponential function. When the arc is congested, its value is 1 , which means that only the first part of the equation is used

$I_{j}=\sum_{i \in E}\left(e^{w_{i}}+e^{b_{i}}\right)$.

We can note that if there are several buses on $\operatorname{arc} a$ (if $b_{i}>1$ ), the term $e^{w i}$ dominant and therefore gives priority to stages with buses; if $b_{i}=0$, the degree of congestion is then taken into account.

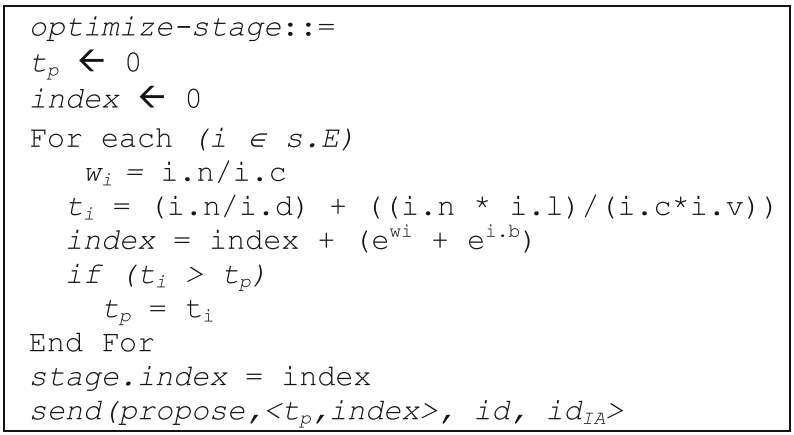

Algorithm 4: Optimization of a stage

\subsection{Bus route agent}

The role of the $B R A$ is to supervise bus agents so as to prevent a local level regulation and the creation of bus queues. In other words, this agent can modify the behavior of bus agents in two different ways: (1) directly: by keeping those buses which are ahead in the plan compared to the preceding ones, at the bus stop for a certain period of time; (2) indirectly: by modifying bus priorities. This agent has a global view of the route it operates on, and can therefore detects bus queues and react to prevent queue formation. This agent has a global view of the route it operates on, and can therefore detects bus queues and react to prevent queue formation. This BRA behavior is important for managing bus regularity and, as we may expect, this will increase passenger satisfaction and therefore service quality.

Internal state of the route agent. The route agent encompasses the following data: (1) the set of arcs traveled by the bus on its route; (2) the set of stops on the route: for each stop, its position, and the distance separating it from the next stop; (3) the set of buses on the route; (4) the frequency of buses introduced onto the route. For two consecutive stops $\mathrm{A}_{i}$ and $\mathrm{A}_{j}$, the route agent maintains the journey time $\mathrm{d}_{i, j}$ of the last bus. This helps to follow the bus journey and to calculate whether the bus is ahead or late compared to the bus immediately preceding it.

Behavior of the route agent. When a bus agent moves to a stop, the time $t$ taken to cover the distance $\mathrm{L}_{i, i-1}$ that separates the two stops $\mathrm{A}_{i}$ and $\mathrm{A}_{i-1}$, is transmitted to the route agent. The route agent then compares $t$ to the time $\left(\mathrm{d}_{i, j}\right)$ taken by the preceding bus and consequently decides whether the bus is ahead or late. The route agent computes the new priority of the bus agent as well as the length of time the bus should wait at the commercial stop if it is ahead.

\section{Experimentation and results}

To test our bimodal control strategy, we have developed a MAS prototype on the JADE ${ }^{3}$ platform (Java Agent Development Framework). JADE offers Java middleware with the overall aim to provide a runtime support for agents.

\footnotetext{
3 jade.tilab.com/
} 


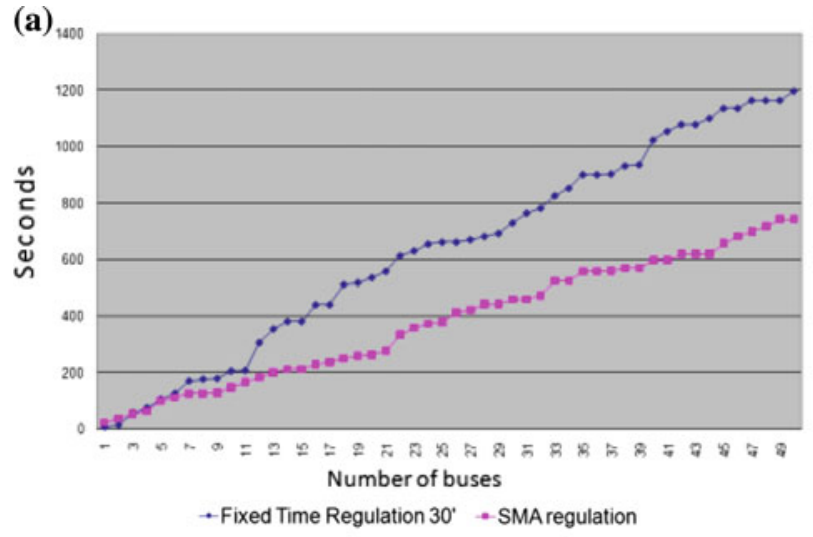

Fig. 4 a Bus cumulated delays. b Private vehicle cumulated delays

We have tested the strategy on a small network of six intersections (Fig. 3a):

- The distance between two adjacent junctions belongs to $[200,400] \mathrm{m}$.

- Each section comprises one or two lanes.

- The saturation flow, which is the maximum exit output of the arcs, is identical for each arc and equal 0.5 vehicle/s.

- At each entry onto the network, we have installed a source that generates private vehicles at a frequency $F \in[4 s \ldots$ $10 s]$.

- Some of the junctions have two stages while others have three stages.

- Two bus routes are considered on the network BR_1 and BR_2. On BR_1, the frequency of generated buses is $80 \mathrm{~s}$ and it is 180 s on BR_2.

We have compared the developed MAS strategy with bus priority to a fixed-time strategy (same duration for all stages) with $30 \mathrm{~s}$ for each stage. We have run the simulation with these two strategies and for half-hour simulation time.

Figure $3 \mathrm{~b}$ depicts bus travel time: the higher curve shows bus travel time between the stops BR1_A1 and BR1_A2 when buses do not request priority at junction $\mathrm{J} 1$; the lowest curve show buses travel time between the two bus stops BR1_A2 and BR1_A3 when buses are asking for priority at junction $\mathrm{J} 2$. We can note that bus travel time improves from a mean of $120 \mathrm{~s}$ to a mean of $60 \mathrm{~s}$ and becomes more regular (flat curve) when bus priority is taken into account.

Figure 4 gives the results of the two strategies for very heavy traffic conditions: Fig. 4a shows the recorded delays for buses with the two control strategies and Fig. 4b shows the same kinds of curves for private vehicles.

These delays correspond to the sum of time lost by all buses (resp. private vehicles) when they stop at traffic lights. As shown in Fig. 4, the MAS strategy improves both traffic of buses and traffic of private vehicles. As we can see, there

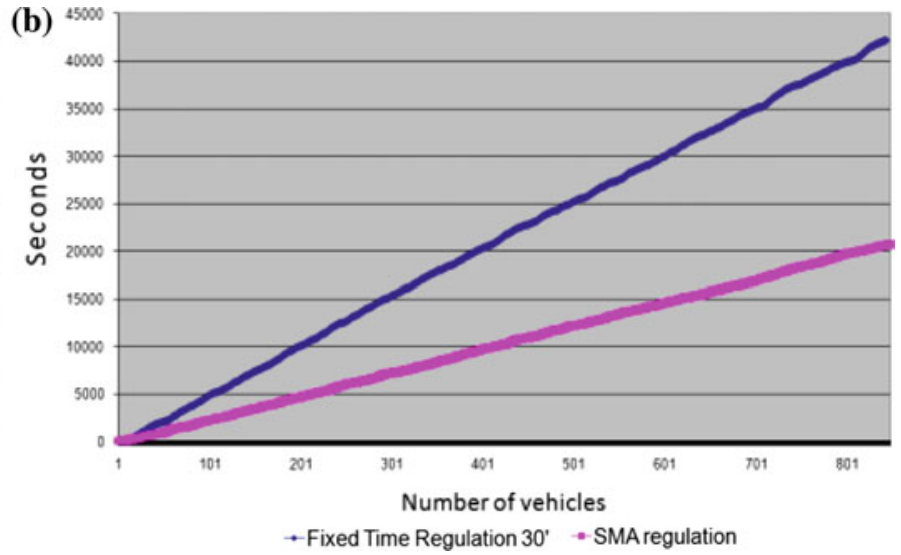

is a decrease of $38 \%$ on lost time spent by buses on traffic light; for the private vehicles, we got a decrease of about $51 \%$. These results can be explained because giving priority to buses decreases traffic jams thus improving private vehicles traffic. These results should be studied in more details: to what point these results are still valid in heavy traffic. It would be interesting to find Pareto front and multi-criteria optimization of total traffic delays and public transport delays.

\section{Conclusion}

In this paper, we have developed a bimodal traffic control strategy based on a MAS. Unlike other approaches, our model takes into account both public transport vehicles such as buses and private vehicle traffic and studies the regulation not only in one intersection but in a whole network. The objective of this research is to improve global traffic, to reduce bus delays and to improve bus regularity in congested areas (keeping regular interval between buses) of the network. We have shown that an agent-based model is well adapted to study complex traffic regulation strategies. In our model, the entities representing the urban network communicate among themselves and negotiate to solve traffic regulating problems. First, we have shown that classical methods of control systems of traffic regulation present several weaknesses: at macroscopic level, they do not take into account mixed traffic and do not allow for the regulation of intervals between buses. At microscopic level computation is time-consuming and therefore cannot be useful for regulating large networks. Secondly, we have presented the multi-agent strategy that computes traffic signal plans based on actual traffic situation and on priority given to buses. Priority is given to buses taking into account intervals between buses. Thirdly, we ran a simulation prototype on the JADE platform. A comparison between bus travel time with and without bus priority 
shows that our control strategy with bur priority improves both travel time and regularity of buses. Our results also show that this bimodal MAS strategy improves conditions of global traffic and reduces bus delays. Additional work, however, is needed: a more realistic network should be defined in the simulation run and more validation and more testing should be undertaken with the definition of several indicators. More precisely, we plan to test our model robustness by varying the model parameters; for example, how traffic volume changes could influence the simulation results, and how these result variations are statically significant.

\section{References}

1. Balbo, F., Pinson, S.: Using intelligent agents for transportation regulation support system design. Transp. Res. Part C Emerg. Technol. 18(1), 140-156 (2010)

2. Balbo, F., Pinson, S.: Dynamic modeling of a disturbance in a multiagent system for traffic regulation. Decis. Support Syst. 41(1), 131$146(2010)$

3. Bazzan, A.L.: Opportunities for multiagent systems and multiagent reinforcement learning in traffic control. Auton. Agents Multi Agent Syst. 18(3), 342-375 (2009)

4. Bhouri, N., Lotito P.: An intermodal traffic control strategy for private vehicle and public transport. In: 10th Euro Working Group on Transportation, Poznan, Poland, pp. 423-428 (2005)

5. Boillot, F., Midenet, S., Pierrelée, J.-C.: The real-time urban traffic control system CRONOS, algorithm and experiments. Transp. Res. Part C 14(1), 18-38 (2006)

6. Camurri, M., Mamei, M., and Zambonelli, F.: "Urban Traffic Control with Co-Fields". In: Weyns, D., Parunak, H.V.D., Michel, F. (eds) Series Lecture Notes in Artificial Intelligence. V. 4389, pp. 239-253. Springer, Berlin (2006)

7. Davidsson, P., Henesey, L., Ramstedt, L.: An analysis of agentbased approaches to transport logistics. Transp. Res. Part C Emerg. Technol. 13(4), 255-271 (2005)

8. De Oliveira, D., Bazzan, A.L., Lesser, V.: Using cooperative mediation to coordinate traffic lights: a case study. In: AAMAS'05, New York, NY, USA, pp. 463-470 (2005)
9. Diakaki, C., Dinopoulou, V., Aboudolas, K., Papageorgiou, M., Ben-Shabat, E., Seider, E., Leibov, A.: Extentions and new applications of the traffic signal control strategy TUC. Transportation Research Record, 1856 (2003)

10. Dion, F., Rakha, H., Zhang, Y.: Evaluation of potential transit signal priority benefits along a fixed-time signalized arterial. J. Transp. Eng. 130(3), 294-303 (2004)

11. Doniec, A., Mandiau, R., Piechowiak, S., Espié, S.: Anticipation based on constraint processing in a multi-agent context. J. Auton. Agents Multi Agent Syst. 17, 339-361 (2008)

12. Ferreira, E.D., Subrahmanian, E., Manstetten, D.: Intelligent agents in decentralized traffic control. Int. IEEE-ITSC'01, Oakland (CA), USA, pp. 705-709 (2001)

13. France, J., Ghorbani, A.: A multiagent system for optimizing urban traffic. In: International conference on IEEE/WIC, Washington, DC, pp. 411-414 (2003)

14. Kachroudi, S., Bhouri, N.: A multimodal traffic responsive strategy using particle swarm optimization. In: 12th IFAC Symposium, Redondo Beach, CA, USA, pp. 13070-13078 (2009)

15. Klügl, F., Rindsfüser, G.: Agent-based route (and mode) choice simulation in real-world networks. In: Intelligent Agent Technology Conference, pp. 22-29 (2011)

16. Mailler, R., Lesser, V: Solving distributed constraint optimization problems using cooperative mediation. In: AAMAS'04. IEEE Computer Society, USA, pp. 438-445 (2004)

17. Mizuno, K., Fukui, Y., Nishihara, S.: Urban traffic signal control based on distributed constraint satisfaction. In: Proceedings of the 41st International Conference on System Sciences, Hawaii, pp. 65 (2008)

18. Roberston, D., Vincent, R.: Bus priority in a network of fixed-time signals, TRRL Repport, No. 666 (1975)

19. Roozemond, D.A.: Using intelligent agents for pro-active, realtime urban intersection control. Eur. J. Oper. Res. 131, 293-301 (2001)

20. Vasirani, M., Ossowski, S.: A computational market for distributed control of urban road traffic systems. IEEE Trans. Intell. Trans. Syst. 12(2), 313-321 (2011)

21. Von Martial, F.: Coordinating plans of autonomous agents. LNCS, no. 610. Springer, Berlin (1992) 